\title{
Disentangling the Effects of Race on Breast Cancer Treatment
}

\author{
Mousumi Banerjee, PhD ${ }^{1}$ \\ Julie George, $\mathrm{ms}^{2,3}$ \\ Cecilia Yee, $\mathrm{ms}^{3}$ \\ William Hryniuk, mo ${ }^{4}$ \\ Kendra Schwartz, MD ${ }^{3,5}$ \\ ${ }^{1}$ Department of Biostatistics, School of Public \\ Health, University of Michigan, Ann Arbor, Michi- \\ gan. \\ ${ }^{2}$ Department of Internal Medicine, Wayne State \\ University, Detroit, Michigan. \\ ${ }^{3}$ Barbara Ann Karmanos Cancer Institute, Wayne \\ State University, Detroit, Michigan. \\ ${ }^{4}$ Cancer Advocacy Coalition of Canada, Toronto, \\ Ontario, Canada. \\ ${ }^{5}$ Department of Family Medicine, Wayne State \\ University, Detroit, Michigan.
}

Supported in part by a grant from the Blue Cross Blue Shield of Michigan Foundation awarded to M.B.

Address for reprints: Mousumi Banerjee, PhD, Department of Biostatistics, School of Public Health, University of Michigan, 1420 Washington Heights, Ann Arbor, Ml 48109-2029; Fax: (734) 936-9582; E-mail: mousumib@umich.edu

Received March 6, 2007; revision received June 5, 2007; accepted June 6, 2007.
BACKGROUND. African Americans (AA) have higher mortality from breast cancer compared with white Americans (WA). Studies using population-based cancer registries have attributed this to disparities in treatment after normalizing the AA and WA populations for differences in disease stage. However, those studies were hampered by lack of comorbidity data and limited information about systemic treatments. The objective of the current study was to investigate racial disparities in breast cancer treatment by conducting a comprehensive medical records review of women who were diagnosed with breast cancer at the Karmanos Cancer Institute (KCI) in Detroit, Michigan.

METHODS. The study cohort consisted of 651 women who were diagnosed with primary breast cancer between 1990 and 1996 at KCI. Multivariable logistic regression analysis controlling for sociodemographic factors, tumor characteristics, comorbidities, and health insurance status was used to assess whether there were differences between WA and AA in the receipt of breast-conserving surgery (BCS), radiation, tamoxifen, and chemotherapy.

RESULTS. There was no significant difference between WA and AA in the receipt of BCS versus mastectomy. Patients with local-stage disease who were enrolled in government insurance plans underwent mastectomy more often (vs BCS plus radiation) compared with patients who were enrolled in nongovernment plans. The rates of receipt of tamoxifen and chemotherapy were similar for local-stage WA and local-stage AA. However, WA were more likely to receive tamoxifen and/ or chemotherapy for regional-stage disease. Married women with regional disease were more likely to receive chemotherapy than nonmarried women.

CONCLUSIONS. The results from this study may be used to target educational interventions to improve the use of adjuvant therapies among AA women who have regional-stage disease. Cancer 2007;110:2169-77. ( 2007 American Cancer Society.

KEYWORDS: racial disparity, treatment, breast cancer, comorbidity, health insurance.

D reast cancer is the most commonly diagnosed malignancy and D is the second leading cause of cancer mortality among women in the United States. Mortality from breast cancer is disproportionately higher in African Americans (AA) compared with white Americans (WA). ${ }^{1-6}$ Several investigators have demonstrated evidence of a calendar period effect in the racial disparity in breast cancer mortality rates: before the late 1970s, there was little difference in mortality rates between AA and WA. ${ }^{7,8}$ The calendar period trends reflect the impact of novel medical interventions, such as screening and treatments, including access to these interventions. AA individuals are diagnosed more often at an advanced stage of disease, primarily because of underutilization of mammographic screening. However, 
residual disparities in breast cancer mortality remain even after adjusting for stage. Another potential explanation is the difference in treatment received by AA women and WA women. Studies of clinical trials and equal-access systems have provided support for the idea that equal treatment will yield similar cancer outcomes between racial/ethnic minorities and WA with similar disease. ${ }^{9-13}$

A few investigators ${ }^{14-18}$ have reported that breast cancer treatment differs between AA women and WA women. However, clear patterns are less discernable when they are examined with respect to clinical, sociodemographic, and insurance designations. Stage of disease alone may not entirely explain disease severity and consequent therapy recommendations. AA women often present with more aggressive breast tumors, including a greater proportion of hormone receptor-negative tumors, poorly differentiated tumors, and high-grade disease. ${ }^{19,20}$ In addition, AA women are more likely to have a diagnosis of 1 or more other comorbid conditions, ${ }^{18,21}$ contraindicating aggressive therapy for their breast cancer. These factors influence physician recommendations for optimal breast cancer therapy, especially systemic adjuvant therapy. There also may be differences in the types of treatment available to AA women compared with WA women because of structural barriers. ${ }^{22}$

Previous studies that relied on cancer registry data from the Surveillance, Epidemiology, and End Results (SEER) Program have suggested the presence of racial disparity in treatment but were hampered by the lack of comorbidity data and the limited information about systemic treatments recorded by hospital-based tumor registries. ${ }^{23,24}$ Analyses of SEER data linked to Medicare claims have delineated treatment use for elderly women and also have suggested a disparity ${ }^{17}$; however, hormone treatments like tamoxifen may be coded inconsistently in Medicare claims. ${ }^{24}$ We undertook a medical records review of women who were diagnosed with early-stage breast cancer at the Karmanos Cancer Institute (KCI), an urban comprehensive cancer center in Detroit that serves a high proportion of poor AA patients. Medical records were obtained from hospitals, outpatient clinics, and private physicians' offices. By using such comprehensive information, we sought to investigate racial disparities in the receipt of optimal breast cancer treatment and to identify the mechanisms that contribute to such disparities.

\section{MATERIALS AND METHODS}

\section{Study Population}

Eligible women were patients who were diagnosed with a first primary, invasive breast cancer between
January 1, 1990, and December 31, 1996, who received their first course of treatment at the Wayne State University-affiliated Harper University Hospital (HUH) and KCI. All women in the analysis were residents of the Detroit metropolitan area at the time of diagnosis, had race classified as AA or WA, and had hospital billing records available for the assessment of health insurance status. The Detroit SEER registry was a source of additional data. For the current analysis, we included women with either local-stage or regional-stage disease as defined by the SEER Program. Local stage includes invasive carcinoma confined to the breast, and regional stage includes invasive carcinoma spread beyond the breast, either by direct extension or to regional lymph nodes. ${ }^{25}$

\section{Study Variables}

Detailed demographic, clinical, pathologic, and treatment information was obtained from the SEER registry, HUH inpatient medical and billing records, and KCI outpatient records. Information was obtained on 1) demographic factors, including age, race, and marital status; 2) comorbidities; 3) tumor characteristics, including stage at diagnosis, tumor size, number of positive lymph nodes, tumor differentiation, and estrogen (ER) and progesterone receptor (PR) status; 4) treatment, including breast conserving surgery (BCS) or mastectomy, radiation therapy, chemotherapy, and/or hormone therapy; and 5) health insurance.

Socioeconomic indicators were determined by geocoding all eligible women based on their SEER-reported addresses at the time of diagnosis and linking these addresses to 1990 United States Census data. The geocoding process first assigned a census block group number based on address to all eligible women. Aggregate socioeconomic status (SES) data specific to each of the census block groups were obtained from the 1990 US Census of Population and Housing Summary Tape File 3A. The SES value for each woman in the study population was computed by using the methodology described and validated by Krieger et al. ${ }^{26}$

A chronic illness index was constructed based on the presence of 5 comorbid conditions abstracted from patients' medical charts, with all conditions given equal weight: 1) body mass index $\geq 30 \mathrm{~kg} / \mathrm{m}^{2}$, 2) diabetes, 3) hypertension, 4) heart disease, and 5) stroke. An index score from 0 to 5 was assigned to each woman and represented the number of comorbid conditions present.

Information on health insurance at the time of breast cancer diagnosis was obtained from hospital billing records and was analyzed as a categorical variable. Nineteen percent of the women were enrolled in a managed care plan at the time of breast 
cancer diagnosis, $31 \%$ were enrolled in a nonmanaged care plan (eg, fee for service [FFS], commercial plans), $42 \%$ were enrolled in Medicare, $7 \%$ were enrolled in Medicaid, and 1\% was self-pay. For analytic purposes, we collapsed managed and nonmanaged care plans into 1 category ("nongovernment insurance"). Medicare and Medicaid were combined to form another category ("government insurance").

Data on surgical treatment type, radiation therapy, and systemic therapy were obtained from combining SEER and medical record review data, counting as "yes," the presence of any treatment in either the SEER data or the patients' charts, with the exception of tamoxifen, which was obtained only from patients' medical records. For analytic purposes, we categorized treatment as primary treatment and adjuvant treatment. Primary treatment was defined as 1) BCS only, 2) BCS plus radiation, 3) mastectomy only, and 4) mastectomy plus radiation. Adjuvant treatment consisted of 1) no systemic therapy, 2) chemotherapy only, 3) tamoxifen only, and 4) both chemotherapy and tamoxifen.

\section{Statistical Methods}

The chi-square test was used to compare the distribution of demographic, socioeconomic, and clinicopathologic variables among AA women and WA women. The Fisher exact test was used when sparse cells were encountered in the contingency tables (ie, expected frequency of any cell $<5$ ). Logistic regression was used to assess variations between WA women and AA women in the type of surgery received, receipt of radiation with BCS, and receipt of tamoxifen and chemotherapy after adjusting for age at diagnosis, marital status, SES, health insurance status, chronic illness index, tumor size, number of positive lymph nodes, tumor differentiation, and ER and PR status. Two-way interactions between variables were tested formally in a logistic regression model. All analyses were stratified by stage at diagnosis (local and regional). Parameter estimates were obtained using the maximum-likelihood method of estimation, and odds ratios (OR) and corresponding 95\% confidence limits (95\% confidence interval [95\% $\mathrm{CI}]$ ) were derived based on the model estimates.

\section{RESULTS}

In total, 651 patients met the eligibility criteria. Among the 651 women, we excluded 9 women because they had a second diagnosis of breast primary within 6 months of the first diagnosis, 3 women who had histology that was categorized as "inflammatory carcinoma" or "phyllodes tumor," 2 women who had no surgery information, and 7 women
TABLE 1

Distribution of Sociodemographic and Tumor Characteristics by Race

\begin{tabular}{|c|c|c|c|c|c|}
\hline \multirow[b]{2}{*}{ Characteristic } & \multicolumn{2}{|c|}{$\begin{array}{l}\text { White } \\
\text { Americans } \\
(\mathrm{n}=242)\end{array}$} & \multicolumn{2}{|c|}{$\begin{array}{l}\text { African } \\
\text { Americans } \\
(\mathrm{n}=388)\end{array}$} & \multirow[b]{2}{*}{$P^{*}$} \\
\hline & No. & $\%$ & No. & $\%$ & \\
\hline Age at diagnosis, y & & & & & .003 \\
\hline$<50$ & 96 & 40 & 111 & 29 & \\
\hline $50-69$ & 106 & 44 & 175 & 45 & \\
\hline$\geq 70$ & 40 & 17 & 102 & 26 & \\
\hline Marital status & & & & & $<.0001$ \\
\hline Married & 159 & 66 & 128 & 33 & \\
\hline Not married & 81 & 33 & 242 & 62 & \\
\hline Unknown & 2 & 1 & 18 & 5 & \\
\hline $\mathrm{SES}^{\dagger}$ & & & & & $<.0001$ \\
\hline Professional & 132 & 55 & 106 & 27 & \\
\hline Working, not poor & 88 & 36 & 49 & 13 & \\
\hline Working, poor & 21 & 9 & 233 & 60 & \\
\hline Insurance plan & & & & & $<.0001$ \\
\hline Government & 94 & 39 & 219 & 56 & \\
\hline Nongovernment & 148 & 61 & 169 & 44 & \\
\hline \multicolumn{6}{|l|}{ Chronic illness index } \\
\hline 0 & 131 & 54 & 74 & 19 & $<.0001$ \\
\hline 1 & 59 & 24 & 140 & 36 & \\
\hline$\geq 2$ & 44 & 18 & 164 & 42 & \\
\hline Missing & 8 & 3 & 10 & 3 & \\
\hline Stage at diagnosis & & & & & .276 \\
\hline Local & 154 & 64 & 230 & 59 & \\
\hline Regional & 88 & 36 & 158 & 41 & \\
\hline Tumor size, $\mathrm{cm}$ & & & & & .010 \\
\hline$<2$ & 131 & 54 & 171 & 44 & \\
\hline$\geq 2$ & 104 & 43 & 209 & 54 & \\
\hline Missing & 7 & 3 & 8 & 2 & \\
\hline No. of positive lymph nodes & & & & & .538 \\
\hline None & 146 & 60 & 224 & 58 & \\
\hline $1-3$ & 60 & 25 & 111 & 29 & \\
\hline$\geq 4$ & 31 & 13 & 44 & 11 & \\
\hline Missing & 5 & 2 & 9 & 2 & \\
\hline Tumor differentiation & & & & & .001 \\
\hline Well/moderate & 109 & 45 & 117 & 30 & \\
\hline Poor & 105 & 43 & 207 & 53 & \\
\hline Missing & 28 & 12 & 64 & 16 & \\
\hline ER status & & & & & .005 \\
\hline Positive & 147 & 61 & 181 & 47 & \\
\hline Negative & 73 & 30 & 149 & 38 & \\
\hline Missing & 22 & 9 & 58 & 15 & \\
\hline PR status & & & & & .001 \\
\hline Positive & 137 & 57 & 158 & 41 & \\
\hline Negative & 81 & 33 & 172 & 44 & \\
\hline Missing & 24 & 10 & 58 & 15 & \\
\hline
\end{tabular}

SES indicates socioeconomic status; ER, estrogen receptor; PR, progesterone receptor. ${ }^{*}$ Chi-square test.

${ }^{\dagger}$ SES was missing for 1 patient.

whose insurance status was "self-pay." Thus, our final dataset contained 630 women who were diagnosed between 1990 and 1996. Of these women, $62 \%$ were $\mathrm{AA}$, and $38 \%$ were $\mathrm{WA}$, reflecting the population served by HUH and KCI in Detroit (Table 1). The AA 
TABLE 2

Treatment Distribution by Race Within Stage

\begin{tabular}{|c|c|c|c|c|}
\hline \multirow[b]{2}{*}{ Treatment } & \multicolumn{2}{|c|}{ Local-stage disease, $\%$} & \multicolumn{2}{|c|}{$\begin{array}{l}\text { Regional-stage } \\
\text { disease, } \%\end{array}$} \\
\hline & $\begin{array}{l}\text { WA } \\
(\mathrm{n}=154)\end{array}$ & $\begin{array}{l}\mathrm{AA} \\
(\mathrm{n}=230)\end{array}$ & $\begin{array}{l}\text { WA } \\
(\mathrm{n}=88))\end{array}$ & $\begin{array}{l}\mathrm{AA} \\
(\mathrm{n}=158)\end{array}$ \\
\hline \multicolumn{5}{|l|}{ Primary treatment } \\
\hline BCS only & 6 & 7 & 0 & 1 \\
\hline Mastectomy only & 39 & 34 & 45 & 49 \\
\hline $\mathrm{BCS}$ and radiation & 44 & 43 & 30 & 23 \\
\hline Mastectomy and radiation & 11 & 15 & 25 & 27 \\
\hline \multicolumn{5}{|l|}{ Adjuvant treatment } \\
\hline None & 23 & 24 & 0 & 11 \\
\hline Chemotherapy only & 19 & 20 & 26 & 40 \\
\hline Tamoxifen only & 38 & 41 & 16 & 25 \\
\hline \multicolumn{5}{|l|}{ Both chemotherapy } \\
\hline and tamoxifen & 14 & 6 & 53 & 20 \\
\hline Missing & 5 & 9 & 5 & 4 \\
\hline
\end{tabular}

WA indicates white Americans; AA, African Americans; BCS, breast-conserving surgery.

women were more likely to be older $(P=.003)$, to be unmarried $(P<.0001)$, to reside in poorer census block groups $(P<.0001)$, to have government insurance $(P<.0001)$, to have more chronic illnesses $(P<$ $.0001)$, to have larger tumors $(P=.01)$, to have more poorly differentiated tumors $(P=.001)$, and to have negative ER tumors $(P=.005)$ and negative $P R$ tumors $(P=.001)$. Table 2 shows the treatment distribution of these patients by race.

There was no significant difference between WA and AA women in the receipt of BCS versus mastectomy for local-stage disease (OR, 0.81; 95\% CI, 0.4$1.65)$ or regional-stage disease $(\mathrm{OR}, 1.65 ; 95 \% \mathrm{CI}$, 0.65-4.16) (Table 3). Furthermore, there was no significant difference between WA and AA women in the receipt of BCS plus radiation versus mastectomy alone (ie, without radiation) for the management of local-stage disease (OR, 0.65; 95\% CI, 0.29-1.47) or regional-stage disease (OR, 1.54; 95\% CI, 0.56-4.25; data not shown). Patients with larger tumors $(\geq 2 \mathrm{~cm}$ ) more frequently underwent mastectomy only versus combined BCS and radiation for local-stage disease than patients with smaller tumors (OR, 2.5; 95\% CI, 1.32-4.76). In addition, patients who were enrolled in government insurance plans more frequently underwent mastectomy only versus combined BCS and radiation for local-stage disease than patients who were enrolled in nongovernment insurance plans (OR, 2.44; 95\% CI, 1.1-5.26).

For local-stage disease, WA women received tamoxifen as frequently as AA women (OR, $0.85 ; 95 \%$ CI, 0.35-2.07) (Table 4). Older women (ages 50-69 years: OR, 2.55; $95 \% \mathrm{CI}, 1.12-5.79$; aged $\geq 70$ years:
OR, 3.88; 95\% CI, 1.23-12.21) and women with positive ER/PR status (OR, 7.92; 95\% CI, 3.88-16.18) were more likely to receive tamoxifen than younger women and women with negative ER/PR status, respectively. For regional-stage disease, WA women received tamoxifen more frequently than AA women (OR, 4.59; 95\% CI, 1.52-13.9). Women aged $>70$ years (OR, 5.58; 95\% CI, 1.23-25.31), women with larger tumors ( $\geq 2 \mathrm{~cm}$ : OR, 4.17 ; 95\% CI, 1.52-11.41), and women with positive ER/PR status (OR, 16.28; 95\% CI, 6.68-39.71) received tamoxifen more frequently than younger women, women with smaller tumors $(<2 \mathrm{~cm})$, and women with ER/PR-negative tumors, respectively.

Among patients with local-stage disease, WA women received chemotherapy as frequently as AA women (OR, 1.3; 95\% CI, 0.52-3.26) (Table 5). Women with positive ER/PR status were less likely to receive chemotherapy than women with negative ER/PR status (OR, 0.21; 95\% CI, 0.1-0.46). Women with larger tumors $(\geq 2 \mathrm{~cm})$ received chemotherapy more frequently than patients with smaller tumors ( $<2 \mathrm{~cm}$; OR, 6.02; 95\% CI, 2.79-12.99). Among patients with regional-stage disease, WA women received chemotherapy more frequently than AA women (OR, 3.1; 95\% CI, 1.09-8.81). Women with positive ER/PR status received chemotherapy less frequently than women with negative ER/PR status (OR, 0.09; 95\% CI, 0.03-0.27). Married women were more likely to receive chemotherapy than nonmarried women (OR, 2.64; 95\% CI, 1.12-6.84).

\section{DISCUSSION}

Similar to previous reports, ${ }^{19,20,27,28}$ in our cancer center-based population, we observed that AA women were more likely to have more advancedstage disease, poorly differentiated tumors, and ER/ PR-negative tumors compared with WA. AA women in our population also more frequently were not married, resided in poor census block groups, had government insurance, and had more chronic illnesses. However, when these factors were taken into account, AA and WA women with local-stage disease were just as likely to undergo BCS compared with mastectomy and to receive adjuvant tamoxifen and chemotherapy. For women with regional-stage breast cancer, there were differences in treatment between AA and WA women, with WA women more frequently receiving tamoxifen and chemotherapy.

Several studies ${ }^{14-18}$ have indicated that breast cancer treatment differs between AA and WA women. However, clear patterns are less discernable when they are examined with respect to racial, socioeco- 
TABLE 3

Unadjusted and Adjusted Odds of Breast-conserving Surgery With or Without Radiation Versus Mastectomy With or Without Radiation by Clinical and Sociodemographic Factors

\begin{tabular}{|c|c|c|c|c|c|c|c|c|}
\hline \multirow[b]{3}{*}{ Variable } & \multicolumn{4}{|c|}{ Local-stage disease } & \multicolumn{4}{|c|}{ Regional-stage disease } \\
\hline & \multicolumn{2}{|c|}{ Unadjusted } & \multicolumn{2}{|c|}{ Adjusted* } & \multicolumn{2}{|c|}{ Unadjusted } & \multicolumn{2}{|c|}{ Adjusted* } \\
\hline & OR & $95 \% \mathrm{CI}$ & OR & $95 \% \mathrm{CI}$ & OR & $95 \% \mathrm{CI}$ & OR & $95 \% \mathrm{CI}$ \\
\hline \multicolumn{9}{|l|}{ Race } \\
\hline WA & 1.05 & $0.64-1.72$ & 0.81 & $0.40-1.65$ & 1.80 & $0.91-3.55$ & 1.65 & $0.65-4.16$ \\
\hline $\mathrm{AA}$ & 1.00 & & 1.00 & & 1.00 & & 1.00 & \\
\hline \multicolumn{9}{|l|}{ ER/PR status } \\
\hline$\geq 1$ Positive & 1.35 & $0.81-2.62$ & 1.00 & $0.55-1.84$ & 1.41 & $0.69-2.90$ & 1.27 & $0.55-2.96$ \\
\hline Both negative & 1.00 & & 1.00 & & 1.00 & & 1.00 & \\
\hline \multicolumn{9}{|l|}{ Tumor size, cm } \\
\hline$<2$ & 1.00 & & 1.00 & & 1.00 & & 1.00 & \\
\hline$\geq 2$ & 0.44 & $0.26-0.72$ & 0.43 & $0.25-0.74$ & 0.41 & $0.20-0.81$ & 0.45 & $0.20-0.98$ \\
\hline \multicolumn{9}{|c|}{ No. of positive lymph nodes } \\
\hline $0-3$ & & & & & 1.00 & & 1.00 & \\
\hline$\geq 4$ & & & & & 0.22 & $0.08-0.60$ & 0.28 & $0.10-0.81$ \\
\hline \multicolumn{9}{|l|}{ Tumor differentiation } \\
\hline Well/moderate & 1.00 & & 1.00 & & 1.00 & & 1.00 & \\
\hline Poor & 0.67 & $0.41-1.11$ & 0.86 & $0.48-1.53$ & 0.44 & $0.22-0.87$ & 0.57 & $0.26-1.28$ \\
\hline \multicolumn{9}{|l|}{ Age at diagnosis, y } \\
\hline$<50$ & 1.00 & & 1.00 & & 1.00 & & 1.00 & \\
\hline $50-69$ & 1.33 & $0.76-2.33$ & 1.87 & $0.92-3.81$ & 0.77 & $0.37-1.61$ & 0.75 & $0.32-1.75$ \\
\hline$\geq 70$ & 1.58 & $0.80-3.13$ & 2.89 & $1.07-7.79$ & 0.46 & $0.16-1.26$ & 0.38 & $0.10-1.49$ \\
\hline \multicolumn{9}{|l|}{ Chronic illness index } \\
\hline 0 & 1.00 & & 1.00 & & 1.00 & & 1.00 & \\
\hline 1 & 1.13 & $0.63-2.06$ & 0.97 & $0.49-1.92$ & 0.98 & $0.45-2.13$ & 1.58 & $0.64-3.94$ \\
\hline$\geq 2$ & 0.75 & $0.41-1.36$ & 0.54 & $0.25-1.15$ & 0.44 & $0.18-1.08$ & 0.68 & $0.23-1.97$ \\
\hline \multicolumn{9}{|l|}{ Marital status } \\
\hline Married & 0.86 & $0.53-1.42$ & 0.72 & $0.40-1.30$ & 1.05 & $0.53-2.05$ & 0.63 & $0.27-1.44$ \\
\hline Not married & 1.00 & & 1.00 & & 1.00 & & 1.00 & \\
\hline \multicolumn{9}{|l|}{ SES } \\
\hline Professional & 1.00 & & 1.00 & & 1.00 & & 1.00 & \\
\hline Working, not poor & 1.08 & $0.57-2.06$ & 1.18 & $0.60-2.34$ & 0.61 & $0.24-1.52$ & 0.60 & $0.22-1.64$ \\
\hline Working, poor & 0.89 & $0.50-1.56$ & 1.00 & $0.48-2.05$ & 0.78 & $0.37-1.67$ & 0.98 & $0.38-2.51$ \\
\hline \multicolumn{9}{|l|}{ Insurance } \\
\hline Government & 0.82 & $0.50-1.33$ & 0.51 & $0.25-1.01$ & 0.57 & $0.28-1.14$ & 0.94 & $0.35-2.50$ \\
\hline Nongovernment & 1.00 & & 1.00 & & 1.00 & & 1.00 & \\
\hline
\end{tabular}

OR indicates odds ratio; 95\% CI, 95\% confidence interval; WA, white American; AA, African American; ER, estrogen receptor; PR, progesterone receptor; SES, socioeconomic status.

${ }^{*}$ ORs from multivariable logistic regression adjusted for race, ER/PR status, tumor size, number of positive lymph nodes (for regional-stage disease only), tumor differentiation, age at diagnosis, chronic illness index, marital status, SES, and insurance status.

nomic, and insurance designations. Several authors have indicated that AA women are less likely to undergo BCS than WA women. ${ }^{14,15}$ However, racial differences in treatment often disappear when factors like SES, hospital size, urban residence, health insurance, and comorbidities are considered. ${ }^{14-16,29}$ The results from a study of a population similar to ours $^{17}$ indicated that AA patients were more likely than WA patients to have no surgery; yet, among women who underwent surgery, AA women were more likely to have BCS than WA women after adjusting for disease stage at diagnosis, SES, and insurance status. Conversely, a recent study of patients with breast cancer at 6 New York City hospitals indicated that minority women had twice the risk of failing to receive radiation therapy after BCS, adjuvant chemotherapy, or hormone therapy. ${ }^{18}$

Concomitant illnesses frequently influence treatment choice. Several authors have reported that comorbidities or contraindicating conditions may lead to deviations from "standard" treatments. For example, in a study by Yancik et al., ${ }^{30}$ certain comorbidities (eg, diabetes requiring insulin, stroke, gastrointestinal tract problems, mental health problems, and a previous malignancy) in breast cancer patients were identified as significant predictors of not receiv- 
TABLE 4

Unadjusted and Adjusted Odds of Receipt of Tamoxifen by Clinical and Sociodemographic Factors

\begin{tabular}{|c|c|c|c|c|c|c|c|c|}
\hline \multirow[b]{3}{*}{ Variable } & \multicolumn{4}{|c|}{ Local-stage disease $(\mathrm{n}=245)$} & \multicolumn{4}{|c|}{ Regional-stage disease $(n=177)$} \\
\hline & \multicolumn{2}{|c|}{ Unadjusted } & \multicolumn{2}{|c|}{ Adjusted* } & \multicolumn{2}{|c|}{ Unadjusted } & \multicolumn{2}{|c|}{ Adjusted* } \\
\hline & OR & $95 \% \mathrm{CI}$ & OR & $95 \% \mathrm{CI}$ & OR & $95 \% \mathrm{CI}$ & OR & $95 \% \mathrm{CI}$ \\
\hline \multicolumn{9}{|l|}{ Race } \\
\hline WA & 1.12 & $0.67-1.85$ & 0.85 & $0.35-2.07$ & 3.42 & $1.76-6.66$ & 4.59 & $1.52-13.90$ \\
\hline $\mathrm{AA}$ & 1.00 & & 1.00 & & 1.00 & & 1.00 & \\
\hline \multicolumn{9}{|l|}{ ER/PR status } \\
\hline$\geq 1$ Positive & 9.91 & $5.27-18.65$ & 7.92 & $3.88-16.18$ & 17.33 & $8.02-37.47$ & 16.28 & $6.68-39.71$ \\
\hline Both negative & 1.00 & & 1.00 & & 1.00 & & 1.00 & \\
\hline \multicolumn{9}{|l|}{ Tumor size, $\mathrm{cm}$} \\
\hline$<2$ & 1.00 & & 1.00 & & 1.00 & & 1.00 & \\
\hline$\geq 2$ & 0.84 & $0.50-1.39$ & 1.73 & $0.89-3.38$ & 1.89 & $1.00-3.58$ & 4.17 & $1.52-11.41$ \\
\hline \multicolumn{9}{|c|}{ No. of positive lymph nodes } \\
\hline $0-3$ & & & & & 1.00 & & 1.00 & \\
\hline$\geq 4$ & & & & & 0.74 & $0.39-1.42$ & 0.63 & $0.24-1.67$ \\
\hline \multicolumn{9}{|l|}{ Tumor differentiation } \\
\hline Well/moderate & 1.00 & & 1.00 & & 1.00 & & 1.00 & \\
\hline Poor & 0.30 & $0.18-0.52$ & 0.41 & $0.21-0.80$ & 0.34 & $0.17-0.67$ & 0.43 & $0.16-1.13$ \\
\hline \multicolumn{9}{|l|}{ Age at diagnosis, y } \\
\hline$<50$ & 1.00 & & 1.00 & & 1.00 & & 1.00 & \\
\hline $50-69$ & 3.43 & $1.88-6.25$ & 2.55 & $1.12-5.79$ & 1.90 & $0.97-3.71$ & 2.40 & $0.86-6.67$ \\
\hline$\geq 70$ & 3.96 & $1.91-8.23$ & 3.88 & $1.23-12.21$ & 2.94 & $1.22-7.04$ & 5.58 & $1.23-25.31$ \\
\hline \multicolumn{9}{|l|}{ Chronic illness index } \\
\hline 0 & 1.00 & & 1.00 & & 1.00 & & 1.00 & \\
\hline 1 & 1.10 & $0.60-2.01$ & 0.78 & $0.35-1.77$ & 0.69 & $0.33-1.43$ & 1.15 & $0.37-3.55$ \\
\hline$\geq 2$ & 1.54 & $0.82-2.87$ & 1.19 & $0.48-2.99$ & 0.78 & $0.37-1.65$ & 0.69 & $0.21-2.24$ \\
\hline \multicolumn{9}{|l|}{ Marital status } \\
\hline Married & 0.92 & $0.56-1.53$ & 0.93 & $0.47-1.84$ & 1.10 & $0.61-2.01$ & 1.29 & $0.51-3.28$ \\
\hline Not married & 1.00 & & 1.00 & & 1.00 & & 1.00 & \\
\hline \multicolumn{9}{|l|}{ SES } \\
\hline Professional & 1.00 & & 1.00 & & 1.00 & & 1.00 & \\
\hline Working, not poor & 0.70 & $0.36-1.36$ & 0.59 & $0.26-1.35$ & 1.17 & $0.53-2.60$ & 1.69 & $0.48-5.94$ \\
\hline Working, poor & 0.65 & $0.36-1.15$ & 0.48 & $0.20-1.15$ & 0.67 & $0.35-1.33$ & 1.16 & $0.41-3.27$ \\
\hline \multicolumn{9}{|l|}{ Insurance } \\
\hline Government & 1.61 & $0.97-2.67$ & 0.90 & $0.40-2.03$ & 1.84 & $1.00-3.39$ & 1.41 & $0.46-4.31$ \\
\hline Nongovernment & 1.00 & & 1.00 & & 1.00 & & 1.00 & \\
\hline
\end{tabular}

OR indicates odds ratio; 95\% CI, 95\% confidence interval; WA, white American; AA, African American; ER, estrogen receptor; PR, progesterone receptor; SES, socioeconomic status.

* ORs from multivariable logistic regression adjusted for race, ER/PR status, tumor size, number of positive lymph nodes (for regional-stage disease only), tumor differentiation, age at diagnosis, chronic illness index, marital status, SES, and insurance status.

ing axillary lymph node dissection among women without obvious advanced disease. Patients with breast cancer who did not receive standard therapy had a median of 3 concomitant medical illnesses compared with 1 concomitant illness for patients who did receive standard therapy. ${ }^{31}$ More comorbid conditions were a significant predictor of underuse of adjuvant breast cancer treatment. ${ }^{18}$ Along with others, we also observed ${ }^{21,29}$ that AA women with breast cancer are more likely to have comorbidities than WA women, which emphasizes the need to adjust for comorbidities to avoid potential confounding of race and comorbidities in assessing treatment disparities. In our adjusted models, comorbidity did not have an independent effect on the receipt of either primary or adjuvant treatment for local- or regional-stage disease.

In our population, AA women with regional-stage breast cancer were less likely to receive adjuvant hormone therapy (tamoxifen) and chemotherapy. Clinical predictors were associated with both of these outcomes, as expected; for example, positive receptor status and greater tumor size were associated with receipt of tamoxifen, and negative receptor status was associated with nonreceipt of chemotherapy. However, in our adjusted models, age $>70$ years pre- 
TABLE 5

Unadjusted and Adjusted Odds of Receipt of Chemotherapy by Clinical and Sociodemographic Factors

\begin{tabular}{|c|c|c|c|c|c|c|c|c|}
\hline \multirow[b]{3}{*}{ Variable } & \multicolumn{4}{|c|}{ Local-stage disease $(\mathrm{n}=255)$} & \multicolumn{4}{|c|}{ Regional-stage disease $(n=180)$} \\
\hline & \multicolumn{2}{|c|}{ Unadjusted } & \multicolumn{2}{|c|}{ Adjusted } & \multicolumn{2}{|c|}{ Unadjusted } & \multicolumn{2}{|c|}{ Adjusted } \\
\hline & OR & $95 \% \mathrm{CI}$ & OR & $95 \% \mathrm{CI}$ & OR & $95 \% \mathrm{CI}$ & OR & $95 \% \mathrm{CI}$ \\
\hline \multicolumn{9}{|l|}{ Race } \\
\hline WA & 1.34 & $0.80-2.24$ & 1.30 & $0.52-3.26$ & 2.67 & $1.30-5.47$ & 3.10 & $1.09-8.81$ \\
\hline $\mathrm{AA}$ & 1.00 & & 1.00 & & 1.00 & & 1.00 & \\
\hline \multicolumn{9}{|l|}{ ER/PR status } \\
\hline$\geq 1$ Positive & 0.19 & $0.11-0.34$ & 0.21 & $0.10-0.46$ & 0.24 & $0.11-0.54$ & 0.09 & $0.03-0.27$ \\
\hline Both negative & 1.00 & & 1.00 & & 1.00 & & 1.00 & \\
\hline \multicolumn{9}{|l|}{ Tumor size, cm } \\
\hline$<2$ & 1.00 & & 1.00 & & 1.00 & & 1.00 & \\
\hline$\geq 2$ & 3.93 & $2.29-6.74$ & 6.02 & $2.79-12.99$ & 0.65 & $0.32-1.33$ & 0.97 & $0.36-2.61$ \\
\hline \multicolumn{9}{|c|}{ No. of positive lymph nodes } \\
\hline $0-3$ & & & & & 1.00 & & 1.00 & \\
\hline$\geq 4$ & & & & & 0.87 & $0.44-1.74$ & 0.68 & $0.24-1.88$ \\
\hline \multicolumn{9}{|l|}{ Tumor differentiation } \\
\hline Well/moderate & 1.00 & & 1.00 & & 1.00 & & 1.00 & \\
\hline Poor & 1.65 & $0.98-2.78$ & 0.65 & $0.30-1.42$ & 0.89 & $0.45-1.76$ & 0.42 & $0.16-1.15$ \\
\hline \multicolumn{9}{|l|}{ Age at diagnosis, y } \\
\hline$<50$ & 1.00 & & 1.00 & & 1.00 & & 1.00 & \\
\hline $50-69$ & 0.13 & $0.07-0.25$ & 0.18 & $0.08-0.41$ & 0.14 & $0.05-0.44$ & 0.07 & $0.02-0.29$ \\
\hline$\geq 70$ & 0.04 & $0.02-0.12$ & 0.10 & $0.03-0.37$ & 0.03 & $0.01-0.11$ & 0.01 & $0.01-0.07$ \\
\hline \multicolumn{9}{|l|}{ Chronic illness index } \\
\hline 0 & 1.00 & & 1.00 & & 1.00 & & 1.00 & \\
\hline 1 & 0.59 & $0.32-1.08$ & 0.69 & $0.28-1.67$ & 0.53 & $0.23-1.20$ & 0.79 & $0.25-2.49$ \\
\hline$\geq 2$ & 0.34 & $0.18-0.66$ & 1.03 & $0.37-2.85$ & 0.44 & $0.19-1.01$ & 2.28 & $0.64-8.12$ \\
\hline \multicolumn{9}{|l|}{ Marital status } \\
\hline Married & 2.14 & $1.27-3.59$ & 1.93 & $0.90-4.15$ & 2.93 & $1.48-5.81$ & 2.64 & $1.12-6.84$ \\
\hline Not married & 1.00 & & 1.00 & & 1.00 & & 1.00 & \\
\hline \multicolumn{9}{|l|}{ SES } \\
\hline Professional & 1.00 & & 1.00 & & 1.00 & & 1.00 & \\
\hline Working, not poor & 1.22 & $0.63-2.37$ & 1.37 & $0.57-3.32$ & 1.51 & $0.62-3.67$ & 1.12 & $0.35-3.60$ \\
\hline Working, poor & 1.03 & $0.57-1.85$ & 1.91 & $0.73-4.99$ & 0.83 & $0.41-1.70$ & 0.81 & $0.28-2.33$ \\
\hline \multicolumn{9}{|l|}{ Insurance } \\
\hline Government & 0.22 & $0.12-0.38$ & 0.44 & $0.18-1.08$ & 0.21 & $0.10-0.42$ & 1.50 & $0.49-4.59$ \\
\hline Nongovernment & 1.00 & & 1.00 & & 1.00 & & 1.00 & \\
\hline
\end{tabular}

OR indicates odds ratio; 95\% CI, 95\% confidence interval; WA, white American; AA, African American; ER, estrogen receptor; PR, progesterone receptor; SES, socioeconomic status.

* ORs from multivariable logistic regression adjusted for race, ER/PR status, tumor size, number of positive lymph nodes (for regional-stage disease only), tumor differentiation, age at diagnosis, chronic illness index, marital status, SES, and insurance status.

dicted the receipt of tamoxifen among women with regional-stage cancer, whereas older age predicted nonreceipt of chemotherapy, and being married increased the odds of receiving chemotherapy. It is noteworthy that, in a study by Bickell et al., ${ }^{18}$ age $>70$ years was not associated with underuse of adjuvant therapy; however, those authors grouped 3 therapies (radiation therapy after BCS, hormone therapy in receptor-positive women, and chemotherapy in receptor negative-women) as a single outcome. Perhaps there were competing effects of age associated with hormone and chemotherapy, like what we observed, leading to a negative association in their study. There is a lack of consensus on the bene- fit of chemotherapy in older women, ${ }^{32,33}$ primarily as a result of underrepresentation of this age group in clinical trials, which may have been a factor in our finding that older age predicted nonreceipt of chemotherapy. Older women more frequently received tamoxifen in our study. One possible explanation for this finding, is the use of tamoxifen as a sole treatment in elderly women, as reported by others. ${ }^{30}$

In several studies, a significant association was observed between marital status and breast cancer treatment. ${ }^{17,33-38}$ Marital status acts as a proxy to social/family support, and it is believed that cancer patients with good family support have healthier lifestyles before and after treatment, improved time to 
diagnosis, and improved treatment decision-making. ${ }^{33,34}$ Silliman et al. $^{39}$ reported that married women with early-stage breast cancer were 2.5 times more likely to receive guideline surgical treatment versus less than guideline surgical treatment compared with unmarried patients after adjusting for comorbidity and physical function. Similarly, Yellen and Cella ${ }^{40}$ reported that adult cancer patients who lived with their children were more willing to undergo aggressive treatments. Blackman et al. ${ }^{38}$ reported that women with breast cancer who had poor family support, including unmarried patients with no living children, were less likely to be prescribed adjuvant tamoxifen for early-stage disease compared with married patients who had living children. In our study, married women were more likely than unmarried women to receive adjuvant chemotherapy for regional-stage disease after adjusting for sociodemographic and tumor characteristics. King et al. ${ }^{37}$ reported similar finding, ie, that chemotherapy is associated with a better marital relationship, suggesting a supportive response from partners. It is worth noting that our populations of AA and WA women differed largely in terms of family structure: Fewer AA patients were married, potentially resulting in fewer AA patients accepting chemotherapy despite having more aggressive tumors because of the lack of a spouse to take care of the family. It should be recognized, however, that AA patients tend to rely on the extended family as a social support network. ${ }^{41}$ Consequently, the importance of marriage to social support may differ between AA women and WA women.

The type of health insurance a woman has can play a role in breast cancer diagnosis and treatment. ${ }^{17,42}$ It has been reported that the treatment of early-stage breast cancer in Health Maintenance Organizations (HMOs) differs from that of local FFS patterns, although not in a consistent manner. ${ }^{42}$ Among elderly patients, it has been reported that the percentage undergoing BCS was similar in HMO and FFS settings, ${ }^{42}$ although the use of BCS reportedly was slightly lower among nonelderly patients in HMOs. In a study of a population similar to ours, women who were insured by Medicaid had a lower likelihood of receiving radiation therapy than women who were not insured by Medicaid. ${ }^{17}$ In our study, patients with local-stage breast cancer who were enrolled in government insurance plans had a lower likelihood of receiving BCS than patients who were enrolled in nongovernment plans. Furthermore, patients who were enrolled in government insurance plans were less likely to be treated with a combination of BCS and radiation (as opposed to mastectomy) for local-stage disease than patients who were enrolled in nongovernment insurance plans. The AA patients in our study largely were enrolled in government insurance plans (56\%) compared with $39 \%$ of WA patients who were enrolled in government insurance plans at the time they were diagnosed with breast cancer.

There are a few possible sources of bias that may influence the generalizability of our findings. First, single-institution studies like ours have drawbacks because of selection bias. Our patient population comprises a large number of older AA women of lower SES with a high incidence of diabetes and obesity. However, it is precisely this patient distribution, together with the large size of our patient cohort, the availability of detailed information regarding demographics, socioeconomic variables, tumor cell characteristics, and chronic illnesses, and especially the treatment information, that allowed us to study a potentially important phenomenon. Second, all of the women who were analyzed in our study had health insurance (ie, they had some access to care) at the time of diagnosis. Thus, we were unable to assess whether differences in access to care among AA and WA women partly may explain treatment disparity. Third, we were unable to measure patient preferences and physician attitudes toward treatment decisions for breast cancer because of the lack of such data in patients' charts.

In summary, in an urban comprehensive cancer center environment, we found that $\mathrm{AA}$ and WA women received similar therapies for local stage cancer. Younger AA women with regional stage cancer were less likely than WA to receive hormone therapy. Older, unmarried AA women were less likely than WA women to receive chemotherapy. This information may be used to target educational interventions to improve the use of adjuvant therapies among AA women with regional stage disease. Strategies that provide increased social and psychological support, both initially, when a decision is being made about choice of therapy, and subsequently, when therapy is administered, may be expected to provide a greater benefit to unmarried individuals.

\section{REFERENCES}

1. American Cancer Society. Cancer Facts and Figures 2006. Atlanta, Ga: American Cancer Society; 2006.

2. Ries LAG, Eisner MP, Kosary CL, et al. eds. SEER Cancer Statistics Review, 1973-1998. Bethesda, Md: National Cancer Institute; 2001.

3. Garfinkel L, Boring CC, Heath CW Jr. Changing trends: an overview of breast cancer incidence and mortality. Cancer. 1994;74:222-227.

4. Russell A, Langlois T, Johnson G, Trentham-Dietz A, Remington P. Increasing gap in breast cancer mortality between black and white women. WMJ. 1999;98:37-39. 
5. Dignam JJ. Differences in breast cancer prognosis among African-American and Caucasian women. CA Cancer J Clin. 2000;50:50-64.

6. Chu KC, Tarone RE, Brawley OW. Breast cancer trends of black women compared with white women. Arch Fam Med. 1999;8:521-528.

7. Jatoi I, Anderson WF, Rao SR, Devesa SS. Breast cancer trends among black and white women in the United States. J Clin Oncol. 2005;23:7836-7841.

8. Brawley OW. Disaggregating the effects of race and poverty on breast cancer outcomes. J Natl Cancer Inst. 2002;94: 471-473.

9. Bach PB, Cramer LD, Warren JL, Begg CB. Racial differences in the treatment of early-stage lung cancer. $N$ Engl $J$ Med. 1999;341:1198-1205.

10. Bach PB, Schrag D, Brawley OW, Galaznik A, Yakren S, Begg CB. Survival of blacks and whites after a cancer diagnosis. JAMA. 2002;287:2106-2113.

11. Yood MU, Johnson CC, Blount A, et al. Race and differences in breast cancer survival in a managed care population. J Natl Cancer Inst. 1999;91:1487-1491.

12. Roach M, Cirrincione C, Budman D, et al. Race and survival from breast cancer: based on Cancer and Leukemia Group B trial 8541. Cancer J Sci Am. 1997;3:107-112.

13. Dignam JJ, Redmond CK, Fisher B, Costantino JP, Edwards BK. Prognosis among African-American women and white women with lymph node negative breast carcinoma: findings from 2 randomized clinical trials of the National Surgical Adjuvant Breast and Bowel Project (NSABP). Cancer. 1997;80:80-90.

14. Muss HB, Hunter CP, Wesley M, et al. Treatment plans for black and white women with stage II node-positive breast cancer. Cancer. 1992;70:2460-2467.

15. Michalski TA, Nattinger AB. The influence of black race and socioeconomic status on the use of breast-conserving surgery for Medicare beneficiaries. Cancer. 1997;79:314319.

16. Velanovich V, Yood MU, Bawle U, et al. Racial differences in the presentation and surgical management of breast cancer. Surgery. 1999;125:375-379.

17. Bradley CJ, Given CW, Roberts C. Race, socioeconomic status, and breast cancer treatment and survival. J Natl Cancer Inst. 2002;94:490-496.

18. Bickell NA, Wang JJ, Oluwole S, et al. Missed opportunities: racial disparities in adjuvant breast cancer treatment. $J$ Clin Oncol. 2006;24:1357-1362.

19. Chen VW, Correa P, Kurman RJ, et al. Histological characteristics of breast carcinoma in blacks and whites. Cancer Epidemiol Biomarkers Prev. 1994;3:127-135.

20. Joslyn SA. Hormone receptors in breast cancer: racial differences in distribution and survival. Breast Cancer Res Treat. 2002;73:45-59.

21. Tammemagi CM, Nerenz D, Neslund-Dudas C, Feldkamp C, Nathanson D. Comorbidity and survival disparities among black and white patients with breast cancer. JAMA. 2005;294:1765-1772.

22. Shavers VL, Brown ML. Racial and ethnic disparities in the receipt of cancer treatment. J Natl Cancer Inst. 2002;94: 334-357.

23. Bickell NA, Chassin MR. Determining the quality of breast cancer care: do tumor registries measure up? Ann Intern Med. 2000;132:705-710.

24. Du XL, Key CR, Osborne C. Community-based assessment of adjuvant hormone therapy in women with breast cancer, 1991-1997. Breast J. 2004;10:433-439.
25. Henson DE, Ries L, Shambaugh EM. Survival results depend on the staging system. Semin Surg Oncol. 1992; 8:57-61.

26. Krieger N, Quesenberry C, Peng T, et al. Social class, race/ ethnicity, and incidence of breast, cervix, colon, lung, and prostate cancer among Asian, black, Hispanic, and white residents of the San Francisco Bay Area, 1988-1992. Cancer Causes Control. 1999;10:525-537.

27. Shavers VL, Harlan LC, Stevens JL. Racial/ethnic variation in clinical presentation, treatment, and survival among breast cancer patients under age 35. Cancer. 2003;97:134-147.

28. Jatoi I, Becher H, Leake CR. Widening disparity in survival between white and African-American patients with breast carcinoma treated in the U.S. Department of Defense Healthcare system. Cancer. 2003;98:894-899.

29. Du W, Simon MS. Racial disparities in treatment and survival of women with stage I-III breast cancer at a large academic medical center in metropolitan Detroit. Breast Cancer Res Treat. 2005;91:243-248.

30. Yancik R, Wesley MN, Ries LA, Havlik RJ, Edwards BK, Yates JW. Effects of age and comorbidity in postmenopausal breast cancer patients aged 55 years and older. JAMA. 2001;285:885-892.

31. Velanovich V, Gabel M, Walker EM, et al. Causes for the undertreatment of elderly breast cancer patients: tailoring treatments to individual patients. J Am Coll Surg. 2002;194: 8-13.

32. Wyld L, Garg DK, Kumar ID, Brown H, Reed MW. Stage and treatment variation with age in postmenopausal women with breast cancer: compliance with guidelines. $\mathrm{Br}$ J Cancer. 2004;90:1486-1491.

33. Kummel S, Elling D, Jeschke S, Schmid P, Thomas A. Chemotherapy treatment options for elderly women with breast cancer. Anticancer Res. 2006;16:1673-1676.

34. Neale AV, Tilley BC, Vernon SW. Marital status, delay in seeking treatment and survival from breast cancer. Soc Sci Med. 1986;23:305-312.

35. Goodwin JS, Hunt WC, Key CR, Samet JM. The effect of marital status on stage, treatment, and survival of cancer patients. JAMA. 1987;258:3125-3130.

36. Du X, Freeman JL, Goodwin JS. The declining use of axillary dissection in patients with early stage breast cancer. Breast Cancer Res Treat. 1999;53:137-144.

37. King MT, Kenny P, Shiell A, Hall J, Boyages J. Quality of life 3 months and 1 year after first treatment for early stage breast cancer: influence of treatment and patient characteristics. Quality Life Res. 2000;9:789-800.

38. Blackman SB, Lash TL, Fink AK, Ganz PA, Silliman RA. Advanced age and adjuvant tamoxifen prescription in early-stage breast carcinoma patients. Cancer. 2002;95: 2465-2472.

39. Silliman RA, Troyan SL, Guadagnoli E, Kaplan SH, Greenfield S. The impact of age, marital status, and physician patient interactions on the care of older women with breast carcinoma. Cancer. 1997;80:1326-1334.

40. Yellen S, Cella DF. Someone to live for: social well-being, parenthood status, and decision-making in oncology. J Clin Oncol. 1995;13:1255-1264.

41. Lincoln KD, Chatters LM, Taylor RJ. Social support, traumatic events, and depressive symptoms among African Americans. J Marriage Fam. 2005;67:754-766.

42. Riley GF, Potosky AL, Klabunde CN, Warren JL, Ballard-Barbash R. Stage at diagnosis and treatment patterns among older women with breast cancer: an HMO and fee-for-service comparison. JAMA. 1999;281:720-726. 